\title{
The Qingdao Twin Registry: A Focus on Chronic Disease Research
}

\author{
Zengchang Pang,' Feng Ning, Jennifer Unger, ${ }^{3}$ C. Anderson Johnson, ${ }^{3}$ Shaojie Wang,' Qian Guo,, \\ Weihua Cao, ${ }^{4}$ and Liming Lee ${ }^{5}$ \\ ' Qingdao Municipal Centers for Disease Control and Prevention, Qingdao, China \\ ${ }^{2}$ Medical School of Qingdao University Qingdao, China \\ ${ }^{3}$ Institute for Health Promotion and Disease Prevention Research and Transdisciplinary Tobacco and Alcohol Use Research Center, \\ University of Southern California Keck School of Medicine, California, United States of America \\ ${ }^{4}$ School of Public Health at Peking University, Beijing, China \\ ${ }^{5}$ Chinese Academy of Medical Sciences and Peking Union Medical College, Beijing, China
}

W ith the changing patterns of morbidity and mortality in China, noncommunicable chronic diseases have become the major threats to the health of the Chinese population. The causes of chronic diseases include genetic factors and behavioral risk factors such as the use of tobacco, alcohol, and other drugs, unhealthy dietary behaviors, and lack of physical activity. Twin studies offer a unique opportunity to disentangle the genetic and environmental risk and protective factors for chronic disease. The Qingdao Twin Registry (QTR) was initiated in 1998 as part of the National Chinese Twin Registry. Over 11,000 pairs of twins and multiples of all ages have been recruited into the registry. Several studies of physical and mental health are underway. Many of the adult twins have completed health and behavioral risk factor surveys, and the adolescent twins are participating in a study of gene-environment interactions in tobacco and alcohol use. Studies of the heritability of personality factors have been conducted. In 2002, Qingdao established the Qingdao Twin Health Promotion Association, a nonprofit organization that supports health services for twins and their parents, organizes special events and health-related activities for twins, and raises funds to conduct twin health examinations. The QTR will be a useful resource for future studies of population genetics in human health and disease.

Twin studies are regarded as one of the better ways to study the relative influences of genes and environment on behavior and disease (Boomsma et al., 2002; Martin et al., 1997; Peltonen, 2003). New advances in the study design and statistical analysis of twin studies have expanded the range of hypotheses that can be tested, including gene-gene and geneenvironment interactions in complex diseases (Boomsma et al., 2002). The main limitations of previous twin studies are small sample sizes and ascertainment bias (Boomsma et al., 2002). Population-based twin registries are valuable for epidemiologic research as they typically have large sample sizes and lack ascertainment bias (Boomsma et al., 2002; Lichtenstein et al., 2002).

Evidence of genetic influences on behavior and health outcomes can be obtained from the classical twin design in which the resemblance of monozygotic (MZ) twins is compared to the resemblance of dizygotic (DZ) twins. For some rare diseases a genetic component of disease vulnerability can be established only if there are sufficient numbers of $\mathrm{MZ}$ and $\mathrm{DZ}$ twin pairs in which at least one member is affected. For such projects, the value of large twin registries is becoming increasingly clear (Boomsma, 1998). Genetic studies of common diseases are complicated by ascertainment bias, population admixture, dichotomization of quantitative disease traits (affected/nonaffected), and inadequate sample size. Systematic, nondisease-based, longitudinal samples circumvent many of these problems and provide the maximum amount of information for meaningful statistical analyses. Twin samples and population cohorts of twins facilitate the identification of disease alleles, making it possible to evaluate the effects of alleles at the population level. They also allow the investigation of the effects of life-course events on health outcomes.

Although twin registries exist in numerous countries throughout the world, until recently relatively little twin research had been conducted in China. China has a lower twin birth rate than many other countries, but the sheer size of its population makes it possible to study very large samples of twins. According to 1990 national vital statistics data, the highest frequency of twins in China was found in

Received 18 May, 2006; accepted 8 August, 2006.

Address for correspondence: Jennifer B. Unger, University of Southern California Institute for Health Promotion and Disease Prevention Research, 1000 S. Fremont, Box 8, Alhambra, CA 91803, USA.E-mail:unger@usc.edu 
Qingdao, in Shandong province. Overall, in China, the majority of twins are MZ although the MZ:DZ ratio varies across regions. The frequency of $D Z$ twins is higher in Northern areas, whereas the frequency of MZ twins is higher in Southern areas. These differences may be caused, in part, by natural status, weather conditions, level of socioeconomic development, and other factors (Gan \& Zheng, 2001).

As part of the Chinese National Twin Registry (CNTR), in 2000, the Qingdao Municipal Centers for Disease Control and Prevention (QDCDC) began to recruit twins as one of four recruitment areas in China. The purpose of the Qingdao Twin Registry (QTR) is to establish a large sample of twins of all ages, including a longitudinal cohort, to investigate the significance of genetic and environmental factors in the incidence of chronic disease, with the ultimate goal of developing medical, behavioral, and environmental interventions to improve the health of the Chinese population. A secondary purpose of the QTR is to conduct outreach to twins through the QDCDC and local news media and involve them in social and health promotion activities. The knowledge gained during this effort can facilitate the formation of twin registries and development of public health programs in other areas of China. This article describes the process of recruiting twins into the registry, the measures obtained from twins sampled from the registry, the results of analyses of QTR data, and the studies currently in progress.

\section{Methods}

Qingdao, China, a city of 7.2 million, is located on the Yellow Sea at the southern tip of Shandong Peninsula. The population of Qingdao is predominantly Han nationality, with minorities comprising only $0.44 \%$ of Qingdao residents. The birth rate is $7.11 \%$ and the mortality rate is $6.70 \%$, yielding an annual population growth rate of $0.41 \%$. Between 1987 and 2002, the rate of twin births was 3.51 per 1000 births. Qingdao has a lower twin birth rate compared with Western nations, but it has the highest twin birth rate in China. Approximately 3000 twin pairs are born in Qingdao each year (Zhou et al., 2005).

Considering the cultural and socioeconomic characteristics of Qingdao and the available infrastructure, several resources and strategies were used to identify twins and recruit them into the QTR. Qingdao has a complete standard immunization registry system for newborns, which registers $95 \%$ of all newborns in Qingdao each year. This registry allows us to count the exact number of twins born each year in Qingdao and to monitor changes in the twin birth rate over time. Known twins and their families were contacted by telephone or in person and asked to provide information to the registry. A media campaign was conducted through the local news media to publicize the QTR and encourage twins to register. To recruit child and adolescent twins, school nurses were asked to register the twins in their schools and provide information about twins who could not be located because they had changed residences (Pang et al., 2003).

\section{Registration Procedure and Initial Adult Data Collection}

From the initial recruitment in 2001, medical records, medical examinations, and questionnaire/interview data were obtained from 1010 twins aged 24 years or older. These twins were scheduled for registration and assessment in pairs on weekend days, to avoid conflict with their work schedules. After providing written informed consent, the twins completed questionnaires and medical examinations and provided a blood sample. Data collection was conducted by trained study interviewers at the QDCDC. Self-administered questionnaire assessment was conducted under study staff supervision. Collection of biological samples (blood) was carried out by a trained medical professional.

\section{Measures}

The medical examination included height, weight, body mass index (BMI), ABO blood typing, and measures of physiological function (e.g., blood pressure, heart rate, electrocardiogram, and metabolism of carbohydrates, lipids, and enzymes). Behavioral and emotional measures included health behaviors (e.g., tobacco and alcohol use), personality factors (e.g., Eysenck 16PF), and psychopathology (e.g., overactive, oppositional, aggressive, withdrawn, depressed, and anxious behavior; sleep and somatic problems). The questionnaire assessment also included demographic information and a standard battery of twin similarity questions, from which a zygosity algorithm was derived.

\section{Ongoing Registration and Data Collection}

Registration of twins continued after the initial data collection. By the end of 2001, 8583 pairs of twins and 62 pairs of triplets had been recruited, including 8783 males and 8383 females. The male-to-female ratio was $1.05: 1$. This represented $0.0159 \%$ of the Qingdao population, including $0.0568 \%$ in some local areas. By 2005, 10,655 pairs of twins had been registered. The age distribution of the registered twins as of 2005 is shown in Table 1.

Efforts are underway to contact samples of twins from the registry and collect more detailed medical and psychosocial information from them. Data have been collected from twins from several districts within Qingdao to make comparisons across socioeconomic strata. In July 2002, we collaborated with the CDC in Zhucheng, a city near Qingdao, to collect data from 1258 Zhucheng twin pairs to compare with the Qingdao twins. In 2004, we collaborated with Jining Medical College to collect data from approximately 1200 pairs of twins in that region. A cohort of twins was created for longitudinal research on chronic diseases and health. The cohort included more than 500 pairs of adult twins (over 24 years old) and more than 300 pairs of children and adolescents (6 to 18 years old). We continue to follow these twins to study changes in their disease incidence and health status over time. A new cohort of 600 adolescent twin pairs 
Table 1

Cumulative Number of Twins Registered in the OTR, 2005

\begin{tabular}{lcc}
\hline Age (years) & Twins (pairs) & Per cent of sample \\
\hline $0-6$ & 3089 & 28.99 \\
$7-14$ & 1095 & 10.28 \\
$15-19$ & 1993 & 18.70 \\
$20-29$ & 1687 & 15.83 \\
$30-39$ & 1325 & 12.44 \\
$40-49$ & 772 & 7.26 \\
$50-59$ & 436 & 4.09 \\
$60+$ & 258 & 2.41 \\
\hline Total & 10,655 & 100.00
\end{tabular}

is currently being created to examine genetic and environmental risk and protective factors for tobacco and alcohol use.

\section{Results}

\section{Rate of Twin Births in Qingdao}

The twin birthrates in Qingdao from 1987 to 2002 are shown in Figure 1. During this time period, the rate of twin births increased from 2 per 1000 births to over 5 per 1000 births. The rate of twin births remained essentially constant from 1987 to 1991, then increased from 1991 to 1998 , then began to decrease again. The twin birthrate during this time period was 2.84 per 1000 births in coastal areas, which was higher than 1.70 per 1000 births in noncoastal areas. The rate of twin births increased more rapidly in countryside and noncoastal areas, which are less socioeconomically developed, than in cities and coastal areas, which are more socioeconomically developed.

\section{Analyses of OTR Survey and Medical Data}

A study of the heritability of personality factors has been conducted with the QTR. This study of 89 twin pairs (55 MZ and $34 \mathrm{DZ}$ ) aged 24 and older investigated Eysenck personality factors, 16PF, and Type A personality. The twin correlation of the Eysenck neuroticism-stability factor $(\mathrm{N})$ was significantly higher among $\mathrm{MZ}$ twins than among DZ twins, yielding a heritability of .45 (Peng et al., 2003). The twin correlations for three of the $16 \mathrm{PF}$ factors also were significantly higher among $\mathrm{MZ}$ twins than among $\mathrm{DZ}$ twins: shy or venturesome $(\mathrm{H})$, self-assured or apprehensive $(\mathrm{O})$, relaxed or tense (Q4). The heritabilities of these factors were $.46, .45$, and .44 , respectively. However, the twin correlation for the forthright or shrewd factor $(\mathrm{P})$ was higher among DZ twins than among $\mathrm{MZ}$ twins, suggesting a more prominent environmental etiology (Wang et al., 2003). Significant agreement in Type A personality was found among MZ twins but not among DZ twins. The concordance rates of Type A personality and hypertension were also significant among $\mathrm{MZ}$ twins but not among DZ twins (Pang, Zhai, et al., 2003). These findings suggest that personality factors such as neuroticism, shyness, self-assurance, relaxation, and Type $\mathrm{A}$ are caused in part by genetic factors.

An analysis of data from the 2001 Qingdao twin survey (Lessov-Schlaggar et al., in press) examined the relative contribution of genetic and environmental influences on cigarette smoking and alcohol drinking among the adult twins in the QTR who participated in the initial data collection ( $n=1010$ individual twins). More than half of the male twins were smokers $(58 \%)$, and $32.5 \%$ reported alcohol consumption. The analyses were restricted to males as the prevalence of tobacco and alcohol use among females was very

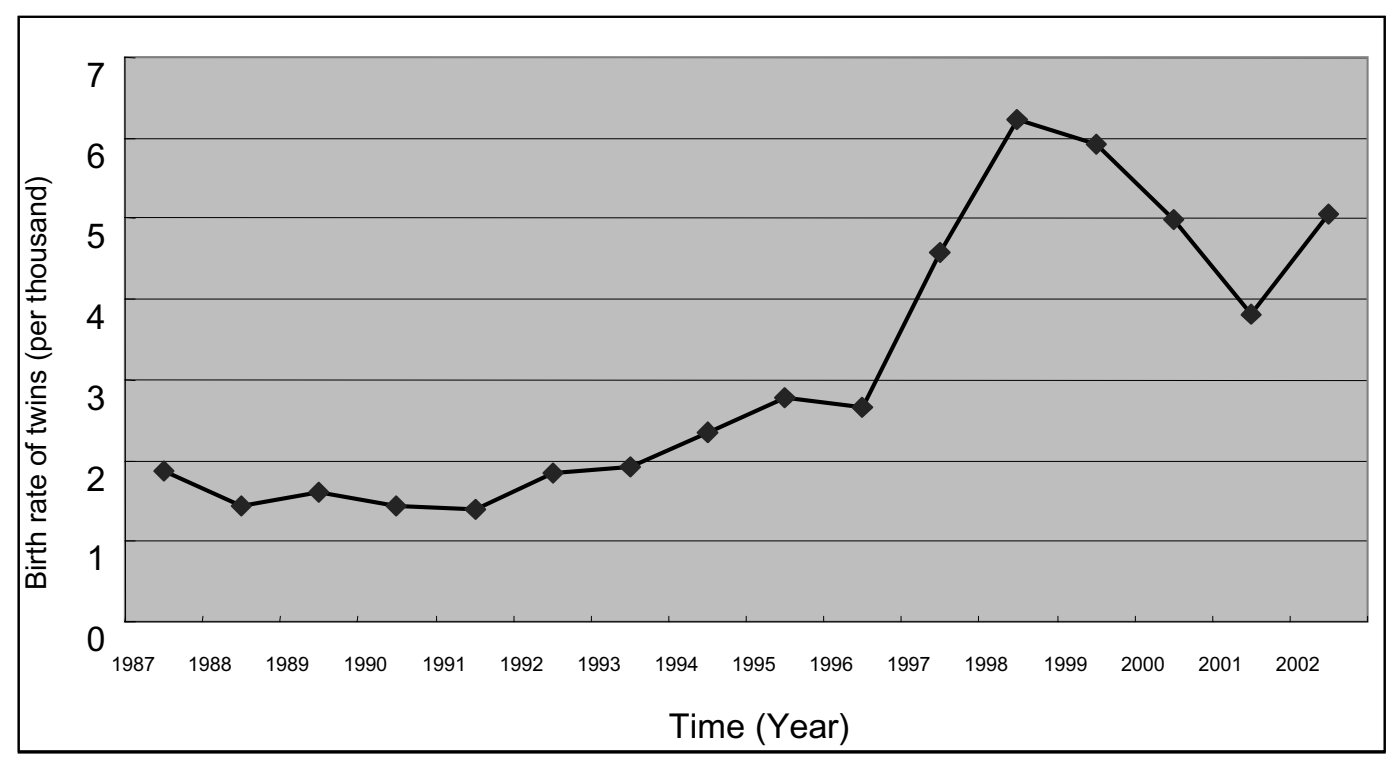

Figure 1

Twin births (per 1000 births) in Qingdao, 1987-2002. 


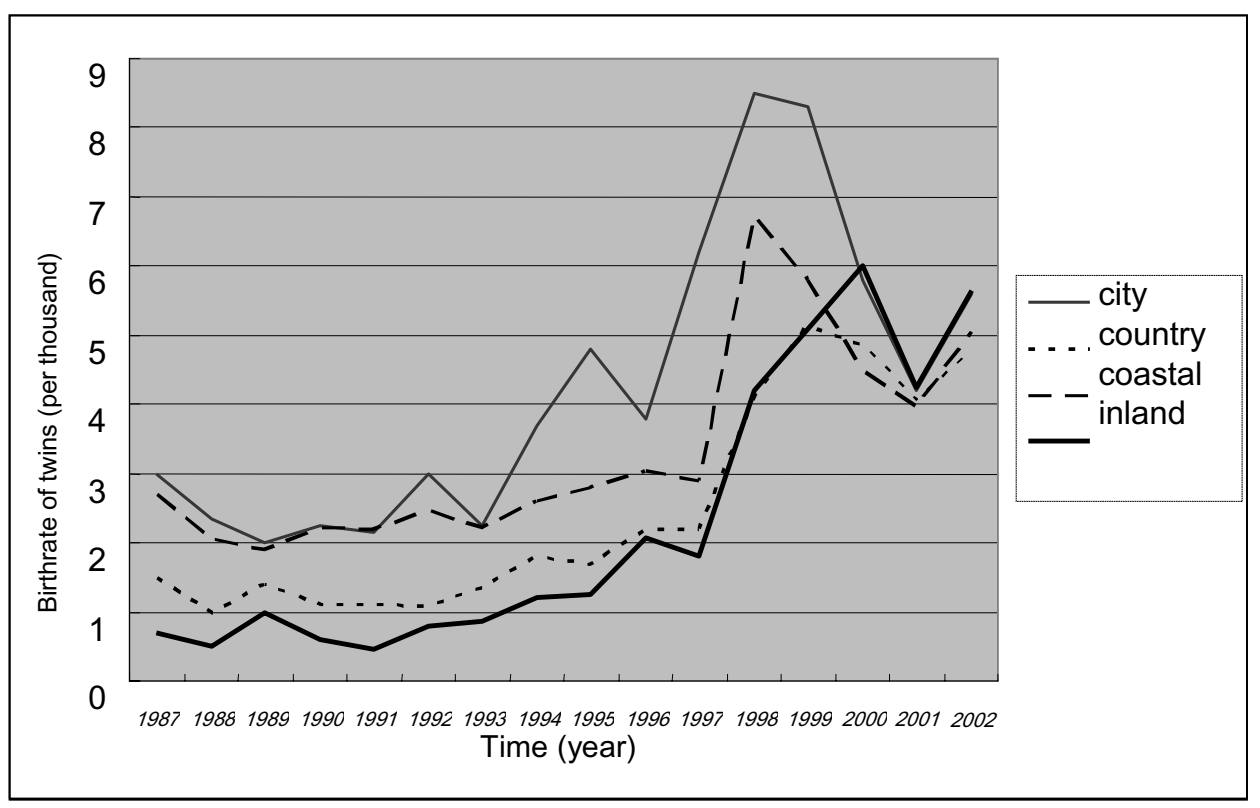

Figure 2

Twin births (per 1000 births) in Qingdao districts, 1987-2002.

low. Among males, current smoking was significantly heritable $(75.1 \%)$ with no evidence for a significant contribution of shared environmental effects. Heavy smoking was more strongly influenced by genes $(66.2 \%)$ than shared environment $(8.7 \%)$. Similarly, current drinking was more strongly influenced by genetic effects $(59.5 \%)$ than by shared environmental effects $(15.3 \%)$. Amount of alcohol consumed was influenced to a similar degree by genetic $(42.4 \%)$ and shared environmental factors $(39.2 \%)$.

\section{Ongoing Research}

In cooperation with the University of Southern California, the QCDC is conducting a study of genetic and environmental risk and protective factors for tobacco and alcohol use among adolescent twins. Six hundred pairs of adolescent twins will complete surveys and health assessments in 2006, including measures of tobacco and alcohol use, peer influences, family cohesion and conflict, stressful life events, depression, hostility, and other related factors. This study will attempt to identify social-environmental protective factors that can lessen the risk of tobacco and alcohol use among adolescents who have genetic predispositions to use tobacco and alcohol. Comparisons will be made with similar age youth drawn from a twin cohort in Southern California (Baker et al., 2002).

\section{Community Outreach: \\ The Oingdao Twin Health Promotion Association}

The Qingdao government and QDCDC established the Qingdao Twin Health Promotion Association in December 2002. This nonprofit organization supports health services for twins and their parents, organizes special events and health-related activities for twins, and raises funds to provide annual medical examinations to the twins. This structure also facilitates the collection of data from the twins and their parents for ongoing studies. The association designated the second Sunday of August of each year as Twins' Day, so that twins have an annual opportunity to celebrate their own holiday with twin reunions, festivals, and performances. QTR also created a website to connect the twins and the research team: http://www.chinatwins.org/

\section{Discussion}

Although twin registries have been established successfully throughout the world, few are based on Chinese populations (Boomsma et al., 2002). Compared to other twin studies, the response rates in the QTR appear to be reasonably good. The recruitment of 10,655 pairs of twins from a city with a total population of 7.2 million indicates that approximately $0.3 \%$ of the entire Qingdao population has been recruited into the twin registry in the first 6 years of its existence. If the rate of twin births was approximately $0.2 \%$ during the time period when most of these twins were born, the Qingdao population would contain approximately 28,800 twins, indicating a participation rate of $74 \%$. Although higher participation rates have been reported in some other twin registries (e.g., 92.5\% in Denmark; Kyvik et al., 1995), the registration of twins in Qingdao is still underway, so the participation rate is likely to increase in the near future. Furthermore, $96 \%$ of the respondents expressed willingness to participate in additional studies. It is also possible to extend twin studies to 
families and the offspring of twins themselves, thus making this cohort even more powerful (Clausson et al., 2000). Ongoing outreach and publicity efforts will be needed to register more twins and their families.

The long-term plan of this program is to establish a population-based twin registry in Qingdao for future studies of specific common complex diseases; conduct detailed phenotyping for clinical and intermediate traits related to cardiovascular diseases; expand studies of twins to families by including their parents, siblings, and offspring for genetic linkage and association studies; and follow up twins in the registry longitudinally. The ultimate goals of the program are health education and promotion, early identification of cases to provide timely medical attention, and the evaluation of long-term effects of identified risk factors. The creators of the QTR wish to develop collaborations with investigators who have expertise in cancer, psychological traits, mental illnesses, and other disease areas (Yang et al., 2002).

The QTR will be a useful resource for large studies on genetic influences on disease incidence and disease outcomes, clinical parameters associated with chronic diseases, aging and age-related health problems, and health behaviors. The combination of survey data with data obtained by linkage to national health related registers enables follow-up studies both of the general twin population and of twins from clinical studies. We have begun to identify genes associated with chronic diseases, health-risk behaviors, and personality traits. This fundamental work will help us to develop improved chronic disease prevention strategies for the general population as well as for Qingdao twins.

\section{Acknowledgments}

This research was supported by the China Medical Board, New York (CMB01, Principal Investigator: Dr Liming Lee) and the University of Southern California Transdisciplinary Tobacco Use Research Center funded by the National Institutes of Health (P50 CA84735-01, Principal Investigator: Dr Carl Anderson Johnson).

\section{$\overline{\text { References }}$}

Baker, L. A., Barton, M., \& Raine, A. (2002). The Southern California Twin Register at the University of Southern California. Twin Research, 5, 456-459.

Boomsma, D., Busjahn, A., \& Peltonen, L. (2002). Classical twin studies and beyond. Nature Reviews. Genetics, 3, 872-882.

Clausson, B., Lichtenstein, P., \& Cnattingius, S. (2000). Genetic influence on birthweight and gestational length determined by studies in offspring of twins. British Journal of Obstetrics and Gynecology, 10, 375-381.
Boomsma, D. I. (1998). Twin registers in Europe: An overview. Twin Research, 1, 34-51.

Gan, J., \& Zheng, J. (2001). Geography factors on Chinese zygosity twins birthrate. Journal of Central China Normal University (Natural Sciences), 35, 3.

Kyvik, K. O., Green, A., \& Beck-Nielsen, H. (1995). The new Danish Twin Register: Establishment and analysis of twining rates. International Journal of Epidemiology, 24, 589-596.

Lessov-Schlaggar, C. N., Pang, Z., Swan, G. E., Guo, Q., Wang, S., Cao, W., Unger, J. B., Johnson, C. A., \& Li, L. (in press). Heritability of cigarette smoking and alcohol use in Chinese male twins: The Qingdao Twin Registry. International Journal of Epidemiology.

Lichtenstein, P., De Faire, U., Flodetus, B., Svedberg, P., \& Pederson, N. L. (2002). The Swedish Twin Registry: A unique resource for clinical, epidemiological and genetic studies. Journal of Internal Medicine, 252, 184-205.

Martin, N., Boomsma, D., \& Machin, G. (1997). A twinpronged attack on complex traits. Nature Genetics, 17, 387-392.

Pang, Z., Bo, T., Wang, S., Peng, X., Zhang, W., \& Zhai, Y. (2003). Analysis on recruitment of mondidymus in Qingdao. Chinese Journal of Public Health, 19, 62-63.

Pang, Z., Zhai, Y., Wang, S., Peng, X., Bo, T., Yan, K., Zhang, Y., Li, X., \& Yuan, M. (2003). Study on relationship of type A personality of twins with hypertension and biochemical index in serum. Chinese Journal of Public Health, 19, 655-656.

Peltonen, L., GenomEUtwin. (2003). GenomEUtwin: A strategy to identify genetic influences on health and disease. Twin Research, 6, 354-360.

Peng, X., Zhai, Y., Wang, S., Pang, Z., Bo, T., Yan, K., Zhang, Y., Li, X., \& Yuan, M. (2003). A twin study on the relationship of Eysenck personality and serum biochemical index. Literature and Information on Preventive Medicine, 9, 129-131.

Wang, S., Pang, Z., Peng, X., Bo, T., Yan, K., Zhang, Y., Zhai, Y., Li, X., \& Yuan, M. (2003). Twin study on the relationship of $16 \mathrm{PF}$ and serum biochemical index. Chinese Journal of Preventive Medicine, 4, 9-11.

Yang, H., Li, X., \& Cao, W. (2002). Chinese National Twin Registry as a resource for genetic epidemiologic studies of common and complex diseases in China. Twin Research, 5, 347-351.

Zhou, L., Li, L., Lu, J., Pang, Z., Hu, Y., Zhan, S., Wang, S., \& Cao, W. (2005). The epidemiologic distribution studies of the twin birth ratios in Qingdao. Chinese Journal of Family Planning, 13, 360-362. 